\title{
ON CORRELATIONS BETWEEN CHARGED AND NEUTRAL PARTICLES IN A CLUSTER MODEL
}

\author{
R. BAIER \\ Department of Theoretical Physics, University of Bielefeld, Germany \\ F.W. BOPP \\ Department of Theoretical Physics, Gesamthochschule Siegen, Germany
}

Received 2 August 1974

(Revised 26 September 1974)

\begin{abstract}
Correlations between charged and neutral pions at high energies are investigated in the framework of a cluster model in which neutral clusters have a decay distribution determined by isospin conservation and statistical independence. For $\left\langle n_{0}\right\rangle_{n}$ an asymptotic expansion around the mean $\left\langle n_{-}\right\rangle$leading asymptotically to a quadratic form in $n_{-}$is derived and compared with data.
\end{abstract}

Recent experiments [1,2] have provided data on correlations between charged and neutral pions in inelastic high-energy scattering and it seems now worthwile to obtain representative fits for the various theoretical concepts.

In this note we study the cluster model [3] in which independently produced neutral clusters [4] decay isotropically in their rest frames. Following the standard picture with factorized momentum and mass dependence of the cluster formation one can write the generating function for the multiplicity distribution simply as

$$
G\left(z_{+}, z_{-}, z_{0}\right)=\exp \left\{\left\langle N_{\mathrm{c}}\right\rangle\left[G^{\mathrm{D}}\left(z_{+}, z_{-}, z_{0}\right)-1\right]\right\},
$$

where $\left\langle N_{\mathrm{c}}\right\rangle$ is the average number of central clusters. $G^{\mathrm{D}}$ is the generating function for the decay, which is obtained after averaging over the cluster masses and which is assumed to be energy independent. To avoid many parameters the presence of the leading particles and the special behaviour of the fragmentation cluster is disregarded.

Existing work [5] which only takes into account a fixed cluster decay multiplicity seems no longer compatible with data and one has to turn to a suitable multiplicity distribution [6]. For this purpose we assume an approximate Poisson distribution for the total cluster decay multiplicities. In order to determine the ratios 
of the various charged states we apply the statistical weights derived by Cerulus [7] for the decay probabilities for isospin zero clusters and obtain the following decay multiplicity distribution

$P_{m_{+}, m_{-}, m_{0}}^{\mathrm{D}}=\delta_{m_{+}, m_{-}} \frac{\lambda^{m_{+}+m_{-}+m_{0}}}{m_{+} ! m_{-} ! m_{0} !} 2^{-m_{+}-m_{-}} \int_{-1}^{+1} \mathrm{~d} x(1+x)^{m_{+}+m_{-}} x^{m_{0}} / N(\lambda)$,

where $\lambda$ is a single parameter describing the size of the clusters and $N(\lambda)$ is the proper normalization. Eq. (2) then leads to the following generating function for the cluster decay*

$$
\begin{aligned}
& G^{\mathrm{D}}\left(z_{+}, z_{-}, z_{0}\right)=\sum_{m_{+}=m_{-}, m_{0}} z_{+}^{m_{+}} z_{-}^{m_{-}} \mathrm{z}_{0}^{m_{0}} P_{m_{+}}^{D}, m_{-}, m_{0} \\
& =\frac{\int_{-1}^{+1} \mathrm{~d} x \mathrm{e}^{z_{0} \lambda x} I_{0}\left[\sqrt{z_{+} z_{-}} \lambda(1+x)\right]}{\int_{-1}^{+1} \mathrm{~d} x \mathrm{e}^{\lambda x} I_{0}[\lambda(1+x)]},
\end{aligned}
$$

where $I_{0}(x)$ is the modified Bessel function.

It is now sufficient to consider correlations between negative and neutral secondaries as the others can be obtained by charge conservation. Applying the appropriate differentiations to eq. (3) we find ${ }^{* *}$

* In eq. (3) an even and odd number of $\pi^{0}$ contribute. One could assume that the clusters have vacuum quantum numbers, but the elimination of the states with negative $G$-parity does not significantly change the following results.

** The correlation integrals between negative pions, $C_{k_{0}}=0, k_{-}$, can be calculated using the multiplication theorem for Bessel function (see for example [8]). It allows one to rewrite the numerator on the right hand side of eq. (3) as

$$
\sum_{k=0}^{\infty} \frac{\left(z_{+} z_{-}-1\right)^{k}}{k !} 2^{-k} \int_{-1}^{+1} \mathrm{~d} x \mathrm{e}^{z_{0} \lambda x}[\lambda(1+x)] k_{I_{k}}[\lambda(1+x)]
$$

Substituting the resulting expression in eq. (1) and applying the derivatives

$$
\left.\left(\mathrm{d} / \mathrm{d} z_{-}\right)^{k} G\left(z_{+}=1, z_{-}, z_{0}=1\right)\right|_{z_{-}}=1
$$

an integral representation for $C_{k_{0}}=0, k_{-}$is obtained. With the help of

$$
\int_{0}^{z} \mathrm{e}^{t} t^{k} I_{k}(t)=\mathrm{e}^{z} z^{k+1}\left[I_{k}(z)-I_{k+1}(z)\right] /(2 k+1)
$$

the final result given in eq. (4) is found. In a similar, slightly more elaborate way the subsequent relations are derived. 


$$
\begin{aligned}
& C_{k_{0}}=0, k_{-}=k=\left\langle N_{\mathrm{c}}\right\rangle \frac{\lambda^{k}}{2 k+1} \frac{I_{k}(2 \lambda)-I_{k+1}(2 \lambda)}{I_{0}(2 \lambda)-I_{1}(2 \lambda)} \\
& C_{k_{0}=}=1, k_{-}=k \\
& \left\langle N_{\mathrm{c}}\right\rangle \lambda^{k} \frac{[2 \lambda /(2 k+3)-\lambda /(2 k+1)] I_{k}(2 \lambda)-[(2 \lambda-1) /(2 k+3)-\lambda /(2 k+1)] I_{k+1}(2 \lambda)}{I_{0}(2 \lambda)-I_{1}(2 \lambda)}, \\
& C_{k_{0}}=2, k_{-}=k=\left\langle N_{\mathrm{c}}\right\rangle \frac{\lambda^{k}}{(2 k+3)(2 k+5)} \\
& \quad \times\left\{\left[4 \lambda+\lambda^{2}((2 k+3)(2 k+5) /(2 k+1)-8)\right] I_{k}(2 \lambda)-\right. \\
& \left.-\left[4(k+1)-2 \lambda(2 k-1)+\lambda^{2}((2 k+3)(2 k+5) /(2 k+1)-8)\right] I_{k+1}(2 \lambda)\right\} \\
& \quad \times\left\{I_{0}(2 \lambda)-I_{1}(2 \lambda)\right\}^{-1} .
\end{aligned}
$$

In order to obtain the parameter $\lambda$ the ratio $C_{-} /\left\langle n_{-}\right\rangle$is used, which is estimated from the proton-proton data [1] above $50 \mathrm{GeV} / c$ as $C_{--} /\left\langle n_{-}\right\rangle \simeq 0.7 \pm 0.1$. In this way $\lambda$ is determined as $\lambda=1.5 \pm 0.1$, which corresponds to an average number of particles per cluster of $\left\langle n_{\text {tot }}\right\rangle^{\mathrm{D}}=2.8 \pm 0.3\left(\left\langle n_{+}\right\rangle^{\mathrm{D}}=\left\langle n_{-}\right\rangle^{\mathrm{D}}=\left\langle n_{0}\right\rangle^{\mathrm{D}}\right)^{*}$, which is in agreement with other estimates [4]. Using this value of $\lambda$ the following asymptotic ratios can be calculated: $C_{--} /\left\langle n_{-}\right\rangle \simeq 0.38 \pm 0.12$ (at present energies not in agreement with experimental data [1]), $C_{-0} /\left\langle n_{-}\right\rangle \simeq 1.1 \pm 0.1$ (not inconsistent with available data [9]), $C_{--0} /\left\langle n_{-}\right\rangle \simeq 0.83 \pm 0.2$ and $C_{00} /\left\langle n_{-}\right\rangle \simeq 1.3 \pm 0.2$ (data not yet available) ${ }^{* *}$. The results yield the following ordering of the two-particle correlation parameters: $C_{+-}>C_{00}>C_{-0}>C_{--}$. Eqs. (4) imply the relation $C_{00}+C_{0_{-}}=C_{--}+C_{+-}$, which is also found (asymptotically) in the framework of other models $[10,11]$.

The interrelation in the production of charged and neutral particles can also be examined with the associated average multiplicity, $\left\langle n_{0}\right\rangle_{n-}$. For the analysis of this quantity it is convenient to exploit the fact, that asymptotic expansions around the mean are applicable, if the correlations are of short range, i.e.

$C_{k}(s) \underset{\bar{s} \rightarrow \infty}{ } \operatorname{ln~s}[12,13]$. To expand $\left\langle n_{0}\right\rangle_{n_{-}}$around $\left\langle n_{-}\right\rangle$we start with the following representation:

* Because of this low value for $\left\langle n_{\text {tot }}\right\rangle^{D}$, the Cerulus coefficients for low multiplicities are the relevant ones for the following results.

** For the ratio $R_{\mathrm{ch}, \mathrm{ch}}(0,0) / R_{\mathrm{o}, \mathrm{ch}}(0,0)$ of the normalized correlation functions at the center in rapidity, $y_{1}=y_{2}=0$, the considered model gives the value $1.08 \pm 0.02$, which is in agreement with ISR data [1]. 


$$
\left\langle n_{0}\right\rangle_{n_{-}} P\left(n_{-}\right)=\frac{1}{2 \pi} \int_{-\pi}^{+\pi} \mathrm{d} t \mathrm{e}^{-i n_{-} t}\left[\sum_{k=0} \frac{C_{1, k}}{k !}\left(\mathrm{e}^{i t}-1\right)^{k}\right] G\left(z_{-}=\mathrm{e}^{i t}, 1,1\right)
$$

and proceed along the method described in ref. [13], where the corresponding expansion for the multiplicity distribution $P\left(n_{-}\right)$is derived. This way one ends up with the asymptotic expansion

$$
\begin{aligned}
& \left\langle n_{0}\right\rangle_{n_{-}}=\left\langle n_{0}\right\rangle+\frac{C_{-0}}{D_{-}} x+\frac{\left(C_{-0}+C_{--0}\right) D_{-}^{2}-C_{-0}\left(C_{---}+3 C_{--}+\left\langle n_{-}\right\rangle\right)}{2 D_{-}^{4}}\left(x^{2}-1\right) \\
& \quad+O(1 / \sqrt{1 n s})
\end{aligned}
$$

where $x=\left(n_{-}-\left\langle n_{-}\right\rangle\right) / D_{-}$and $D_{-}^{2}=\left\langle n_{-}^{2}\right\rangle-\left\langle n_{-}\right\rangle^{2}$. The coefficients can be calculated using eq. (4) and the result is shown in fig. 1 . Satisfactory agreement with the data $[2,9]$ is found. The negative coefficient of $x^{2}$ implies a downward curvature. Re-

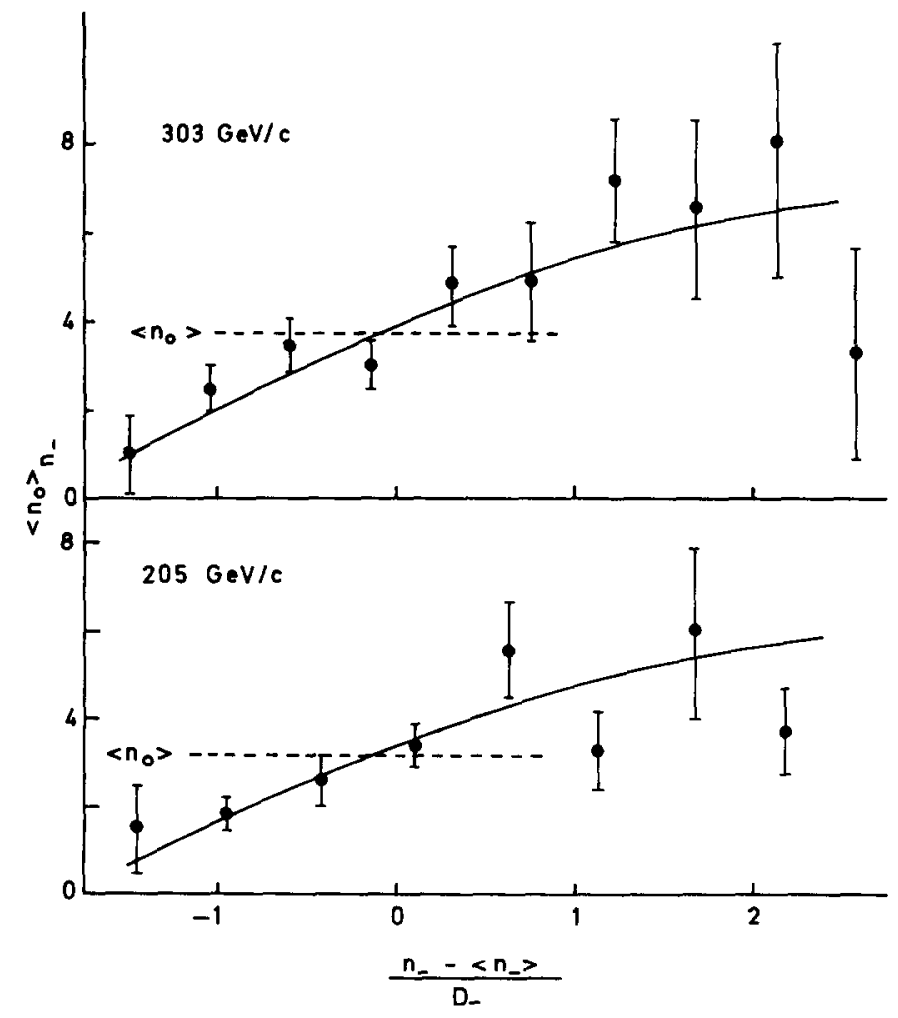

Fig. 1. $\left\langle n_{0}\right\rangle n_{-}$as a function of $\left(n_{-}-\left\langle n_{-}\right\rangle\right) D_{-}$. Data are form ref. [9], the solid curve shows the results from eq. 6 . 
writing the above expansion (6) in powers of $n_{-}\left(\left\langle n_{0}\right\rangle_{n_{-}}=a_{0}+b_{0} n_{-}+c_{0} n_{-}^{2}\right)$ one obtains an asymptotically constant slope of

$$
b_{0}=\frac{C_{-0}}{D_{-}^{2}}-\frac{\left(C_{-0}+C_{--0}\right) D_{-}^{2}-C_{-0}\left(C_{---}+3 C_{--}+\left\langle n_{-}\right\rangle\right)}{D_{-}^{6}} \simeq 0.67 \pm 0.03,
$$

which is also in good agreement with fitted values at the highest energies available $\left(b_{0}=0.69 \pm 0.15\right.$ at $305 \mathrm{GeV} / c$ and $0.62 \pm 0.14$ at $1500 \mathrm{GeV} / c$ [9]); $c_{0}$ vanishes asymptotically.

Interchanging charged and uncharged an equivalent expansion for $\left\langle n_{-}\right\rangle_{n_{0}}$ is obtained. The slope parameter is predicted to be $b_{-} \simeq 0.75 b_{\mathrm{o}}$.

Similar relations can be derived for the higher associated correlations. Interesting experimentally might be $\left(C_{00}\right)_{n_{-}}$. We find for the leading term at $n_{-}=\left\langle n_{-}\right\rangle$the expression $\left(C_{00}-\left(C_{-0}\right)^{2} / D_{-}^{2}\right) \simeq 0.56\left\langle n_{-}\right\rangle$.

The purpose of this paper is to show that a model with isospin-zero clusters indeed reproduces the main features of the high energy correlation data. But because of the present experimental errors and as "asymptotia" is not yet reached many models $[2,3,10,14]$ are able to explain this set of data.

\section{References}

[1] L. Foà, Proc. of the 2nd Int. Conf. on elementary particles, Aix-en-Provence, J. de Phys. 34 (1973) C1-317.

[2] A. Wróblewski, Invited talk at the 4th Conf. on hadrodynamics, Pavia, Warsaw University preprint IFD/73/9 (1973).

[3] A. Bialas, Invited talk at the IVth Conf. on hadrodynamics, Pavia, CERN preprint TH-1745 (1973), and references therein.

[4] S. Pokorski and L. Van Hove, Acta Phys. Pol. B5 (1974) 229.

[5] A. Arneodo and J. Kubar-Andre, Correlations between neutral and charged pions in multiparticle production, Université de Nice preprint N TH 74/1 (January 1974), and references therein.

[6] F. Csikor, I. Montvay and F. Niedermayer, Phys. Letters B49 (1974) 47 ,

[7] F. Cerulus, Nuovo Cimento 19 (1961) 528.

[8] M. Abramowitz and I.A. Stegum, Handbook of mathematical functions (Dover Publications, New York) Ch. 9.

[9] F.T. Dao and I. Whitmore, Phys. Letters B46 (1973) 252.

[10] G.H. Thomas and B. Webber, Phys. Rev. D9 (1974) 3113.

[11] J. Engels, K. Fabricius and K. Schilling, Bielefeld University preprint 74/03 (1974).

[12] D.K. Campbell and S.-J. Chang, Phys. Rev. D8 (1973) 2996;

Y. Tomozawa, Phys. Rev. D8 (1973) 2138.

[13] R. Baier and F. Widder, Nuovo Cimento 20A (1974) 260.

[14] P. Grassberger and H.I. Miettinen, CERN preprints, ref. TH 1870 and 1896 (1974). 